\title{
Drosophila Netrin-B controls mushroom body axon extension and regulates courtship-associated learning and memory of a Drosophila fragile $\mathrm{X}$ syndrome model
}

\author{
Huaixing Kang ${ }^{1}$, Juan Zhao ${ }^{1}$, Xuan Jiang ${ }^{1}$, Guangxu Li ${ }^{1}$, Wen Huang ${ }^{1}$, Huili Cheng ${ }^{2^{*}}$ and Ranhui Duan ${ }^{1,3,4^{*}}$
}

\begin{abstract}
Mushroom body (MB) is a prominent structure essential for olfactory learning and memory in the Drosophila brain. The development of the MB involves the appropriate guidance of axon lobes and sister axon branches. Appropriate guidance that accurately shapes MB development requires the integration of various guidance cues provided by a series of cell types, which guide axons to reach their final positions within the MB neuropils. Netrins are axonal guidance molecules that are conserved regulators of embryonic nerve cord patterning. However, whether they contribute to MB morphogenesis has not yet been evaluated. Here, we find that Netrin-B (NetB) is highly expressed in the MB lobes, regulating lobe length through genetic interactions with the receptors Frazzled and Uncoordinated-5 from $24 \mathrm{~h}$ after pupal formation onwards. We observe that overexpression of NetB causes severe $\beta$ lobe fusion in the $M B$, which is similar to the MB defects seen in the Drosophila model of fragile $X$ syndrome (FXS). Our results further show that fragile-X mental retardation protein FMRP inhibits the translational activity of human ortholog Netrin-1 (NTN1). Knock-down of NetB significantly rescues the MB defects and ameliorates deficits in the learning and memory in FXS model Drosophila. These results indicate a critical role for NetB in MB lobe extension and identify NetB as a novel target of FMRP which contributes to learning and memory.
\end{abstract}

Keywords: Mushroom body (MB), Netrin-B (NetB), Axon extension, Fragile X syndrome (FXS)

\section{Introduction}

To form a complete and functional nervous system, neurons need to extend axons to reach specific targets. Proper axon guidance and extension are controlled by neuronal cell surface receptors and their extracellular ligands, known as axon guidance molecules [1-3]. The mushroom body (MB) neuropils in Drosophila melanogaster are a powerful model system for investigating axonal guidance and extension because of the unique structure of the MB [4-7]. During development, MB neurons, called Kenyon cells, experience a sequential differentiation process into three neuronal sub-types: $\alpha / \beta$ neurons, $\alpha^{\prime} / \beta^{\prime}$ neurons and $\gamma$ neurons. The cell bodies

\footnotetext{
*Correspondence: 2556266023@qq.com; duanranhui@sklmg.edu.cn

${ }^{2}$ Changchun Children' Hospital, Changchun 130000, Jilin, China

${ }^{1}$ Center for Medical Genetics, School of Life Sciences, Central South University,

Changsha 410078, Hunan, China

Full list of author information is available at the end of the article
}

of these MB neurons form a pair of quadruple clusters in the dorsal posterior cortex and project their axons through an axon tract called the peduncle to the anterior region. The axons bifurcate into two branches at the anterior end of the peduncle and segregate into medial $\left(\beta, \beta^{\prime}\right.$ and $\left.\gamma\right)$ and dorsal $\left(\alpha, \alpha^{\prime}\right)$ lobes. Many axonal guidance cues are known to regulate development of the $\mathrm{MB}$ lobes or even sister branch-specific development. For example, Robo $2 / 3$ signaling mainly regulates dorsal and medial lobe extension, while Sema-1a signaling directs lobe outgrowth and orientation in a lobe- and axon branch-specific manner. Likewise, Eph signaling guides specific axon branches of MB neurons [3, 4, 6, 8]. As essential chemotropic cues for axon guidance during neural development, netrins are also expressed in the MBs [3], but their function in $\mathrm{MB}$ development is not yet clear.

(c) The Author(s). 2019 Open Access This article is distributed under the terms of the Creative Commons Attribution 4.0 International License (http://creativecommons.org/licenses/by/4.0/), which permits unrestricted use, distribution, and reproduction in any medium, provided you give appropriate credit to the original author(s) and the source, provide a link to the Creative Commons license, and indicate if changes were made. The Creative Commons Public Domain Dedication waiver (http://creativecommons.org/publicdomain/zero/1.0/) applies to the data made available in this article, unless otherwise stated. 
Netrins are a family of laminin-related proteins that function as chemotropic guidance cues for migrating cells and axons during neural development [9, 10]. In Drosophila, two Netrin homologs have been identified, known as NetA and NetB. As key axonal guidance molecules, detailed studies have been conducted to investigate their roles in ventral nerve cord (VNC) development. Both NetA and NetB are highly expressed by the cells of the VNC midline, mainly guiding commissural axons either toward or away from the midline. In Drosophila, Frazzled (Fra), Uncoordinated-5 (Unc-5) and Down syndrome cell adhesion molecule (Dscam) have been identified as the three main receptors of netrins. During VNC development, Fra is involved in the attraction of commissural axon towards the midline, while Unc-5 acts to prevent commissural axons from crossing the midline. Dscam serves as an attractive receptor which acts in parallel with Fra during VNC development, but it can also respond to multiple other as-yet-unidentified ligands [11-14]. During MB development, Dscam is required for $\mathrm{MB}$ axon sister branch segregation [15-17], but it is less clear whether Fra and Unc-5 regulate $\mathrm{MB}$ development.

As a prominent structure for higher-order functions in the Drosophila protocerebrum, the $\mathrm{MB}$ is essential for learning and memory, similar to the hippocampus in mammals [3, 18]. Drosophila with MB defects exhibit impaired memory formation and deficits in sleep homeostasis. Fly models of cognitive disorders, such as fragile X syndrome (FXS) or Alzheimer's disease, or of inherited cognitive deficits, for example caused by mutation of $Z C 3 H 14$, show subtle MB defects [18-27]. FXS, the most common form of inherited monogenic disorder caused by mutation of the fragile- $X$ mental retardation 1 (Fmr1) gene, leads to transcriptional silencing of its encoded fragile-X mental retardation protein FMRP [28-30]. In $d f m r 1$ mutant flies, the MB is characterized by the failure of the $\beta$ lobes to stop at the brain midline. Research by both McBride et al. and Chang et al. has demonstrated that $\mathrm{MB}$ defects can be rescued and courtship activity and associated memory can be restored by treatment with metabotropic glutamate receptor (mGluR) antagonists or GABAergic inhibitors [23-25]. These findings highlight the importance of structural integrity of the MB to learning and memory.

In our study, we focused on the roles of netrins in Drosophila MB development. By loss- and gain-of-function experiments we demonstrate that MB axons display lobe-specific NetB signaling, regulating lobe axon outgrowth. Fra and Unc5 were found to participate in lobe extension via genetic interaction with NetB signaling. Overexpression of $N e t B$ results in severe $\beta$ lobe fusion similar to the MB abnormality observed in $d f m r 1 \mathrm{mu}-$ tant models, with increased NetB protein levels observed in the brains of $d f m r 1$ mutants. We further report that NetB human ortholog NTN1 mRNA physically interacts with FMRP, and that NTN1 mRNAs exhibit abnormal polyribosome profiles. Importantly, MB and memory defects are ameliorated in FXS Drosophila by knock-down of $N e t B$.

\section{Materials and methods \\ Drosophila stocks}

Flies were cultured on standard Drosophila yeast-cornmeal molasses food at $25^{\circ} \mathrm{C}$. The following RNAi lines were obtained from the Vienna Drosophila Resource Center for use as stocks: UAS-NetB-RNAi (VDRC330183), UAS-NetA-RNAi (VDRC108577), and UAS-Dscam1-RNAi (VDRC108835). The following lines were obtained from the Bloomington Drosophila Stock Center: $\operatorname{Net}^{\Delta}{ }^{\Delta}$ (BDSC66878), NetB ${ }^{\Delta}$ (BDSC66879), NetB $^{\text {tm }} \quad$ (BDSC66880), Dscam1 ${ }^{1}$ (BDSC5934), Fra ${ }^{3}$ (BDSC8813), Unc-5 ${ }^{\text {MIO4273 }}$ (BDSC37426), UAS-Fra-RNAi (BDSC40826), UAS- Unc-5-RNAi (BDSC33756), UAS- $m C D 8-G F P$ (BDSC5130), dfmr $1^{\Delta 3}, d f m r 1^{50 M}$ along with Elav-Gal4, OK107-Gal4, repo-Gal4 and other balancer flies. UAS-NetA and UAS-NetB lines were constructed and the expression level of NetA and NetB were verified.

\section{Antibodies and immunodetection}

For indirect immunofluorescent staining, larval and adults brains were dissected, fixed and stained as previous described [31]. The following antibody dilutions were used: monoclonal 1D4 antibody (anti-FasII), 1:20 (Developmental Studies Hybridoma Bank, Iowa city, IA, USA); c-myc (9E10, anti-c-myc), 1:20 (Santa Cruz, TX, USA); Cy3-labeled anti-mouse, 1:200 (Jackson Immunoresearch, PA, USA). Confocal imaging was performed using a confocal microscope (TCS SP5; Leica Microsystems, Wetzlar, Germany). Defects in lobe length and width were defined by visually inspecting all brains. Only obvious and unambiguous differences in length or width were considered $[3,5]$.

For western blotting (WB), the protein quantity of each sample was estimated by the Bradford assay (Thermo Fisher Scientific, MA, USA), before being subjected to SDS-PAGE. The primary antibodies were diluted as follows: c-myc (9E10), 1:50 (Santa Cruz, TX, USA); $\beta$-actin, $1 \mu \mathrm{g} / \mathrm{ml}$ (Abcam, UK), anti-FMRP (1C3): 1:500 (Millipore, MA, USA).

\section{RNA immunoprecipitation (RNA-IP)}

Immunoprecipitation was performed according to the modified protocol of Gross et al. [32]. Lysates from HEK293 cells for RIP were prepared in lysis buffer (20 $\mathrm{mM}$ Tris- $\mathrm{HCl}, \mathrm{pH} 7.5 ; 150 \mathrm{mM} \mathrm{NaCl} ; 5 \mathrm{mM} \mathrm{MgCl}_{2} ; 1$ mM DTT; and 1\% Triton X-100, supplemented with 
proteinase and RNase inhibitors) on ice. The solution was then precleared with $180 \mu \mathrm{l}$ Dynabeads protein G (Thermo Fisher Scientific, MA, USA) for $2.5 \mathrm{~h}$. Next, one third of the supernatant was saved as the input fraction for WB. The rest of the supernatant was then incubated with antibody-bound Dynabeads Protein G, blocked beforehand with anti-FMRP antibody (1C3) or normal mouse IgGs, overnight at $4{ }^{\circ} \mathrm{C}$. Then, one sixth of the beads were boiled prior to WB. Total RNA from the remaining beads was extracted with an equal amount of $C$. elegans RNA added into the reaction mix. The RNA extract underwent reverse transcription, followed by quantitative real-time PCR (RT-qPCR). Relative values were calculated using the ddCt method with $18 \mathrm{~S}$ rRNA from C. elegans as an external control gene.

\section{Liner sucrose gradient fraction}

Cultured normal and fmr1 mutant lymphoblastoid cells were incubated with cycloheximide $(100 \mu \mathrm{g} / \mathrm{ml})$ or 30 $\mathrm{mM}$ EDTA at $37^{\circ} \mathrm{C}$ for $30 \mathrm{~min}$ to arrest polyribosome migration. Cells were lysed using lysis buffer $(10 \mathrm{mM}$ HEPES-KOH, pH 7.5; $150 \mathrm{mM} \mathrm{KCl;} 10 \mathrm{mM} \mathrm{MgCl}_{2} ; 1$ $\mathrm{mM}$ DTT; $100 \mu \mathrm{g} / \mathrm{mL}$ cycloheximide; and $1 \%$ Triton $\mathrm{X}-100$, supplemented with proteinase and RNase inhibitors). Cytoplasmic extracts were loaded on a $15-60 \%$ (wt/vol) sucrose gradient and centrifuged at 45,000 rpm in a Beckman SW-55 rotor (Beckman Coulter, CA, USA) for $60 \mathrm{~min}$ at $4{ }^{\circ} \mathrm{C}$ to separate them into 10 fractions. Each fraction was isolated and analyzed by RT-qPCR [33], with an equal amount of C. elegans RNA used as an external control.

\section{Behavioral training and testing}

The behavioral training and testing were performed as described previously [24, 25]. Virgin male flies were collected within $3 \mathrm{~h}$ of eclosion. Each male fly was collected in an individual food tube. Virgin W1118 females were collected and kept in food tubes in groups of 10 . Flies were aged for $5 \mathrm{~d}$ in a $12 / 12 \mathrm{~h}$ light/dark cycle at $25^{\circ} \mathrm{C}$ before behavioral training and testing. All testing was performed between $11 \mathrm{am}$ and $3 \mathrm{pm}$ during the relative light phase with comfortable humidity. Mated females were $5 \mathrm{~d}$ old and were observed to mate with a male the night before training. All male flies were transferred to fresh food tubes the day before testing. Male flies were assigned randomly to groups for behavioral training and testing in a polystyrene chamber $(8 \times 10 \times 12 \mathrm{~mm})$, with the behavioral training and testing performed blind to group [24, 34, 35]. The total amount of time that a male spent on courtship activity when paired with an unanesthetized female target was scored either during a test period of $10 \mathrm{~min}$ or until successful copulation. The courtship index (CI) was measured as the percentage of total time spent courting during the observation [34].

\section{Pavlovian olfactory learning and memory}

Flies were trained with the classical conditioning procedure from Tully and Quinn [36]. After one training cycle, the olfactory learning ( 3 min-memory) was tested. The experiments were carried out at $25^{\circ} \mathrm{C}$ and $70 \%$ humidity. Flies were collected after eclosion and incubated for 5 days before testing learning.

\section{Statistical methods}

To assess lobe length, samples were obtained, with experimenters blind to genotype. Each $\alpha / \beta$ lobe of a $\mathrm{MB}$ was categorized into "normal", "short" and "overextended" classes. For "normal", the MBs show a paired neuropil structure in which the $\alpha$ lobes project toward the dorsal surface, while the $\beta$ and $\gamma$ lobes project toward the midline of the brain. For "short", the MBs exhibit obviously reduced or absent lobes. "Overextended $\alpha$ lobes" is defined as overextension of the tip of the dorsal lobes toward the interhemispheric region, "overextended $\beta$ lobes" as the $\beta$ lobes cross the MB midline and fused, and "overextended $\gamma$ lobes" as $\gamma$ lobes project dorsally outside the normal axis due to overextension $[6,8]$. The phenotypic severity in each group was quantified as the percentage of MBs that belong to the defective class, statistical significance was assessed by Fisher's exact test. ANOVAs were performed for comparison between multiple groups. The other data were analyzed using Student's two-tailed $t$-test.

\section{Results}

\section{Drosophila NetB regulates MB lobe extension}

To investigate the roles of Drosophila netrins in MB development, we assessed the overall structure of the adult $\mathrm{MB}$ (dissected at $3 \mathrm{~d}$ after eclosion) in wild type (WT), Net $A$ deletion mutant $N e t A^{\Delta}$, and NetB deletion mutant $N e t B^{\Delta}$ flies. Anti-Fasciclin (FasII) antibody labels $\alpha / \beta$ lobes strongly and $\gamma$ lobes weakly in wild type (WT) strain W1118 adult brains (Fig. 1a). The WT MB showed a paired neuropil structure in which the $\alpha$ lobes project toward the dorsal surface, while the $\beta$ and $\gamma$ lobes project toward the midline of the brain. No obvious defects were observed in $N e t A^{\Delta}$ flies compared with WT flies. However, the stereotyped morphology of MB lobes was disrupted, leading to a relatively smaller $\mathrm{MB}$ structure with short lobes in $N e t B^{\Delta}$ flies (Fig. 1a). We statistically analyzed the MB defects, focusing on $\alpha / \beta$ lobes, which are easy to visualize. The $\alpha / \beta$ lobes were significantly shorter in $N e t B^{\Delta}$ flies compared to those in WT flies, with the percentage of short $\alpha$ and $\beta$ lobes being 33 and $23 \%$ in the two groups, respectively (Fig. $1 b, p<0.01$ ). We also documented the effects of NetB on $\alpha, \beta^{\prime}$ and $\gamma$ lobe length (Additional file 1: Table S1).

To further analyze how netrins regulate MB morphogenesis, we investigated the effects of knock-down and 


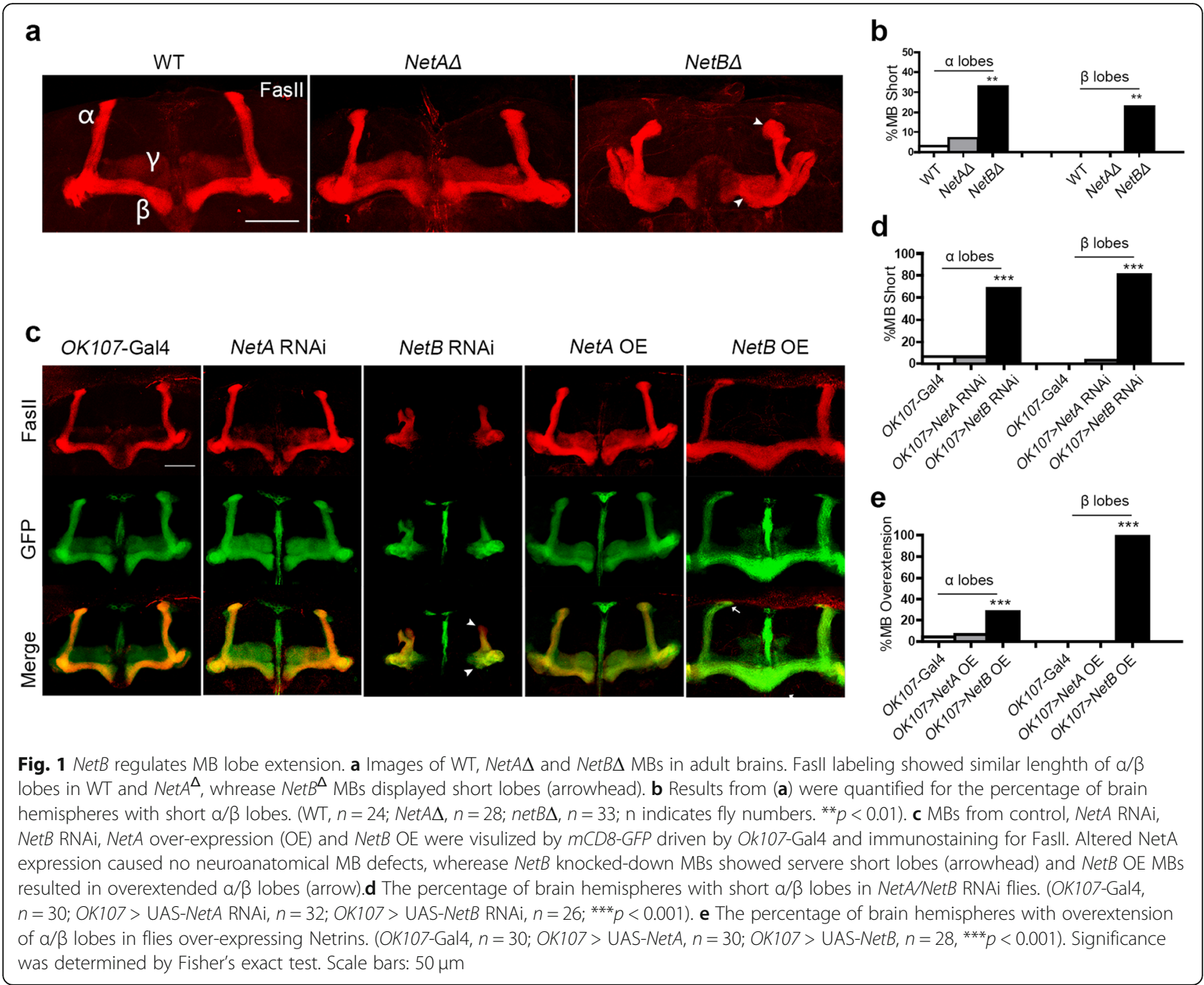

overexpression of NetA and NetB using the pan-MB neuroblast driver OK107-Gal4. Reductions or increases of NetA activity resulted in no obvious neuroanatomical $\mathrm{MB}$ defects compared with control. Knock-down of $N e t B$ in $\mathrm{MB}$ neurons resulted in significantly greater numbers of short lobes, reminiscent of the phenotype observed in $N e t B^{\Delta}$ mutants. Overexpression of NetB resulted in slight overextension of the tip of the $\alpha$ lobe toward the interhemispheric region and severe fusion in the $\beta$ lobe (Fig. 1c, d). Similar phenotypes were also observed using the pan-neuronal elav-Gal4 driver (Additional file 2: Figure S1). These results suggest that $N e t B$ can regulate the precise extension of MB lobes.

\section{Net $B$ is highly expressed in MB and controls MB lobe morphogenesis from $24 \mathrm{~h}$ after pupal formation}

Next, we examined the expression pattern of $\mathrm{NetB}$ during Drosophila brain development. We labeled a myc-tagged membrane-tethered version of $\operatorname{NetB}\left(N e t B^{\mathrm{tm}}\right)$, expressed under the control of the endogenous Netrin promoter
$[37,38]$. We observed that during the larval period and early pupal stage, $N e t B$ was mainly expressed in the ventral midline (Fig. 2a, b). From $24 \mathrm{~h}$ after pupal formation (APF), NetB was expressed in the ellipsoid body (EB) and around the end of peduncles (Fig. 2c). $N e t B$ was highly expressed in the MB lobes from $36 \mathrm{~h}$ APF into adulthood (Fig. 2d-f).

During $\mathrm{MB}$ development, both $\gamma$ and $\alpha^{\prime} / \beta^{\prime}$ neurons are generated and acquire similar projection patterns during the instar stage. After pupal formation, $\gamma$ neurons undergo a dramatic reorganization and become restricted to the end of peduncle at around $24 \mathrm{~h} \mathrm{APF}$, while $\alpha^{\prime} / \beta^{\prime}$ neurons remain relatively unchanged. Meanwhile, all MB neurons which are born after pupal formation become $\alpha / \beta$ neurons, and their axons extend to form the adult-specific lobes [39]. To determine the stage at which axonal defects start to be observed, we analyzed the morphology of MB lobes in NetB RNAi flies during metamorphosis. NetB RNAi lobes were indistinguishable from control at larval stage and at $18 \mathrm{~h}$ 

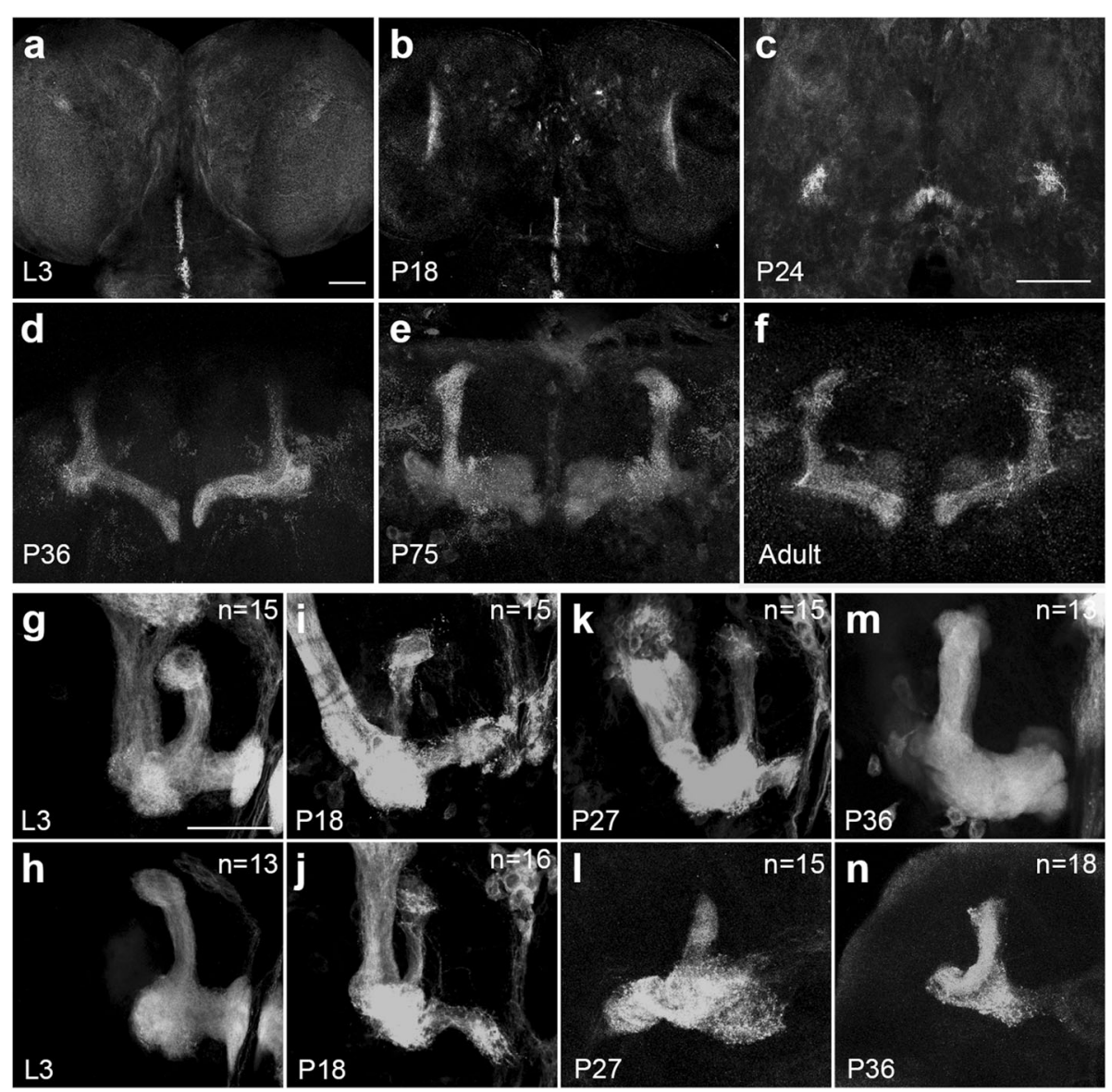

Fig. 2 NetB expression pattern and MB phenotypes in NetB RNAi flies during brain development. a-f NetB expression pattern in NetB ${ }^{\text {tm }}$ flies, as revealed by anti-myc staining (gray). a-b Strong expression of NetB was observed in the VNC cells at third Larva stage (L3) and $18 \mathrm{~h} \mathrm{APF.} \mathbf{c}$ At stage of the $24 \mathrm{~h} \mathrm{APF}$, NetB was enriched around the end of peduncles of MB and in the ellipsoid body (EB). $\mathbf{d}-\mathbf{f}$ NetB expression from $36 \mathrm{~h} \mathrm{APF}$ until adult stage. MB lobes were strongly stained with anti-myc. g-n The MB morphology of the control (OK107> UAS-mCD8-GFP) and NetB RNAi (UAS-NetB RNAi/+; UAS-mCD8-GFP/+;OK107-Gal4/+)flies at L3,18h APF,27 h APF,36 h APF. Upper line shows the control group MBs and lower line shows the NetB RNAi MBs. Numbers in the upper right corners indicate the numbers of observed MBs in corresponding genotypes

APF (Fig. $2 \mathrm{~g}-\mathrm{j} ; n=15$ and $n=13$ for control and NetB RNAi larval MBs, respectively; $n=15$ and $n=16$ for control and NetB RNAi $18 \mathrm{~h}$ APF MBs, respectively). Notably, at $27 \mathrm{~h}$ APF and $36 \mathrm{~h}$ APF, NetB RNAi MBs showed a much greater proportion of short lobes (Fig. $2 \mathrm{k}-\mathrm{n} ; n=$ 15 and 15 for control and NetB RNAi 27 h APF MBs, respectively; $n=13$ and 18 for control and NetB RNAi 36 h APF MBs, respectively). These results imply that NetB is required for the $\mathrm{MB}$ lobe projection to mature after the completion of degeneration.

Receptors Fra and Unc-5 are involved in MB development through genetic interaction with NetB

Dscam, Fra, and Unc-5 have been shown to function as netrin receptors during VNC development in Drosophila $[11,13,40]$. We investigated the possible roles of these receptors in $\mathrm{MB}$ formation. We made use of RNAi constructs targeting each receptor which were driven by
elav-Gal4. Knock-down of Dscam1 resulted in very thin and faint $\alpha / \beta$ lobes, but had no effect on lobe length $(n=30)$. Knock-down of Fra led to a significantly greater proportion of short $\alpha$ lobes, but no $\beta$ lobe defects $(n=28)$. After knock-down of Unc-5, the dorsal or medial lobes were partially or completely lost compared with control $(n=34)$ (Fig. 3a, b). Furthermore, we also investigated the $\mathrm{MB}$ phenotypes of the receptor heterozygous mutant alleles (the mutants are often recessive lethal). Removing one copy of these receptors was not sufficient to cause any neuroanatomical defects in the MB on its own. Our data broadly indicate roles for Dscam in regulating divergent segregation of axons in the MB, Fra in $\alpha$ lobe outgrowth, and Unc-5 in both $\alpha$ and $\beta$ lobe extension.

We then examined whether NetB signaling affecting MB morphogenesis is dependent on these receptors. As overexpression of $N e t B$ results in overextended lobes, 

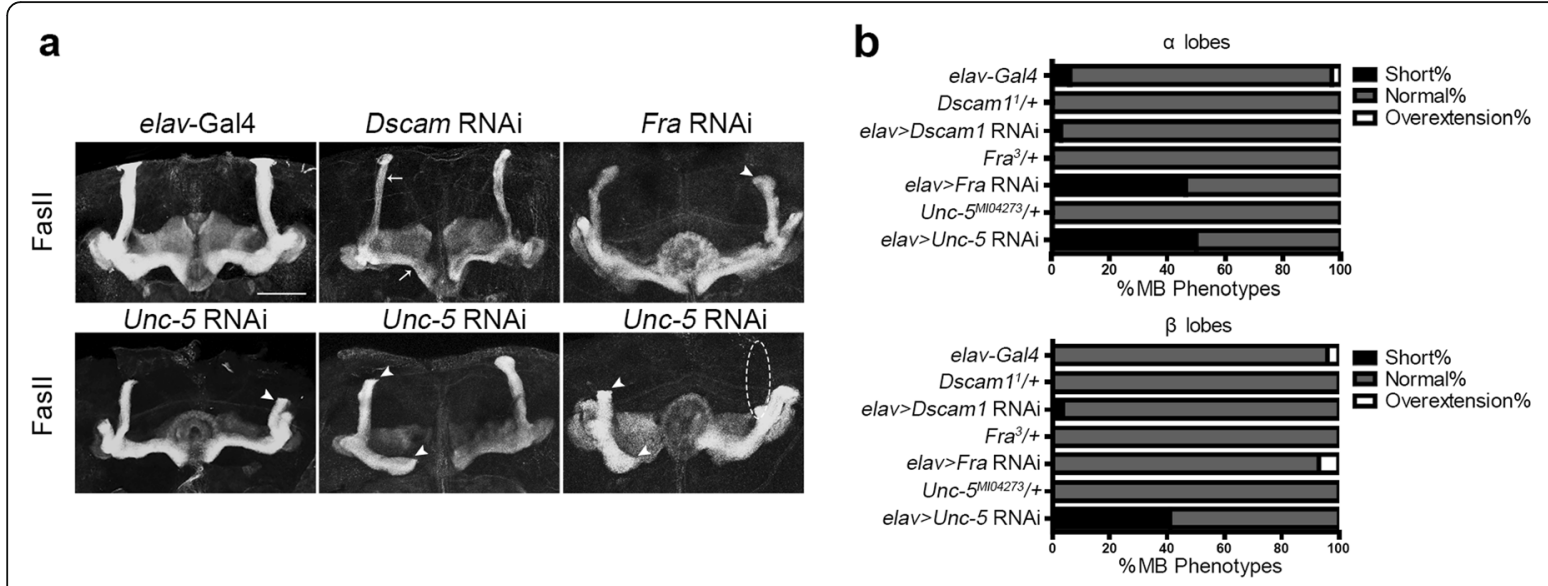

C
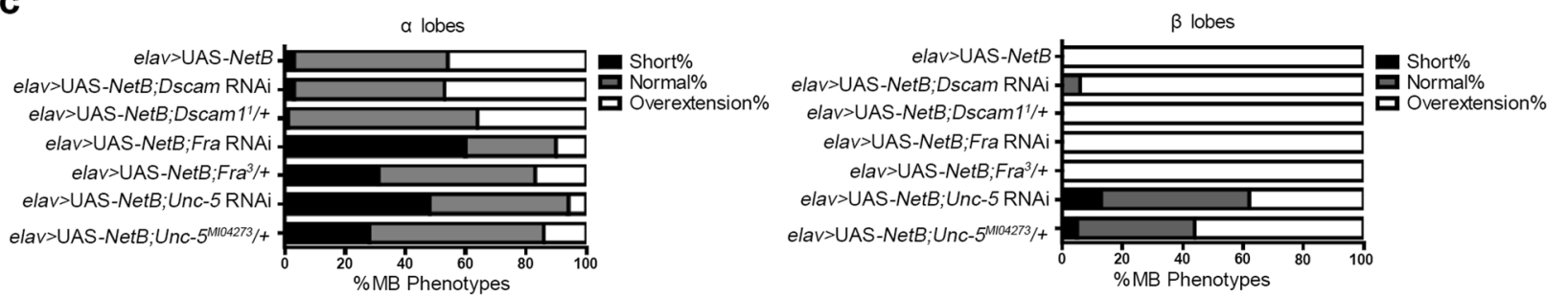

Fig. 3 Receptors Fra and Unc-5 show genetic interaction with NetB in MB lobe extension. a Receptors Dscam, Fra and Unc-5 are involved in MB development. Dscam RNAi MBs displayed thin a/ $\beta$ lobes with normal length compared with control MBs; Fra RNAi MBs displayed short a lobes (arrowhead); Knock-down of Unc-5 caused both short (arrowhead) or even missing (dashed lines) $\alpha / \beta$ lobes. $\mathbf{b}$ The percentage of brain hemispheres with short a/ $\beta$ lobes (elav-Gal4, $n=30$; elav > Dscam RNAi, $n=30$; elav > Fra RNAi, $n=28$; elav $>$ Unc-5 RNAi, $n=34 ;{ }^{* * *} p<0.001 ;$ Dscam $1^{1} /+, n=25$;

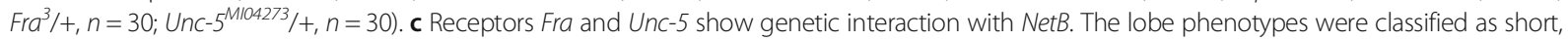
normal or overextension, and the quantification was shown as the percentages of brain hemispheres in each category. The percentage of overextended a lobes was significantly reduced in elav > UAS-NetB; Unc-5 RNAi (or Unc-5 ${ }^{\text {M104273/+ }}$ ) and in elav > UAS-NetB; Fra RNAi (or $\mathrm{Fra}^{3 /+}$ ) compared with NetB OE (elav > UAS-NetB); The percentage of overextended $\beta$ lobes was significantly reduced only in elav > UAS-NetB;

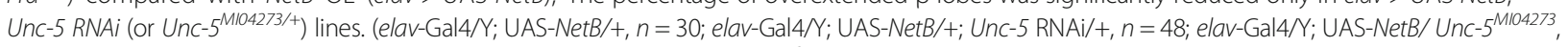
$n=36$; elav-Gal4/Y; UAS-NetB/+; FraRNAi/+, $n=24$; elav-Gal4/Y; UAS-NetB/Fra ${ }^{3}, n=36 ;$ elav-Gal4/Y; UAS-NetB/+; /Dscam RNAi, $n=30 ;$ elav-Gal4/Y; UAS-NetB/Dscam 1 ${ }^{1}, n=34 ; p<0.0001$ ). Significance was determined by Fisher's exact test. Scale bars: $50 \mu \mathrm{m}$

disruption of the NetB pathway may reduce these defects. We investigated the genetic interaction between netrin receptors and $N e t B$ by combining either a Dscam $1^{1}, \mathrm{Fra}^{3}$ or $\mathrm{Unc}_{-} 5^{\mathrm{MIOH273}}$ allele (or an RNAi construct against one of these receptors) with elav-Gal4driven NetB overexpression. Potential genetic interaction was quantified as the percentage of MB lobes categorized as short, normal, or overextended. We did not observe any significant alterations to the NetB overexpression phenotype when $N e t B$ overexpression was combined with mutations (or RNAi knock-down) in Dscam. Loss of one copy (or RNAi-mediated knock-down) of Fra reduced the percentage of $\alpha$ lobe overextension with no alteration to fused $\beta$ lobe morphology. Introduction of $U n c-5^{M I O 4273}$ (or RNAi-mediated knock-down of Unc-5) led to a robust decrease in both $\alpha$ and $\beta$ lobe extension (Fig. 3c). Furthermore, we examined MB phenotypes in $\mathrm{NetB}^{\Delta}$ homozygotes in combination with heterozygous mutations of receptor Fra and Unc-5. Introduction of these heterozygous alleles did not aggravate the phenotypes of $N e t B$ mutants, implying that
$N e t B$ is the primary ligand responsible for regulating $\mathrm{MB}$ extension via Fra and Unc-5 (Table 1).

\section{Genetic reduction of NetB rescues MB defects and ameliorates the learning and memory in $d f m r 1$ mutants} As a result of the deletion of dFMRP, $d f m r 1$ mutant flies exhibit deficits in cognitive function associated with a relatively high frequency of $\beta$ lobes which cross the midline [24, 25, 41]. In our study, NetB-overexpressing flies exhibited similar defects as dfmrl mutants. We

Table 1 No significant alteration of the NetB ${ }^{\Delta}$ phenotypes in combination with mutations in Fra and Unc-5 (Fisher's exact test)

\begin{tabular}{|c|c|c|c|}
\hline Genotypes & \#brains & Lobe & \% Short \\
\hline \multirow[t]{2}{*}{$\mathrm{Net}^{\Delta} / \mathrm{Y}$} & 32 & $a$ & 33 \\
\hline & & $\beta$ & 18 \\
\hline \multirow[t]{2}{*}{$\mathrm{NetB}^{\Delta} / Y_{;} \mathrm{Fra}^{3} /+$} & 25 & $a$ & 32 \\
\hline & & $\beta$ & 14 \\
\hline \multirow[t]{2}{*}{$\mathrm{NetB}^{\Delta} / \mathrm{Y} ;$ Unc-5 $5^{\mathrm{Ml04273}} /+$} & 26 & $a$ & 33 \\
\hline & & $\beta$ & 15 \\
\hline
\end{tabular}


wondered whether $\mathrm{MB}$ defects are influenced by the NetB signaling pathway in $d f m r 1$ mutants. The severity and variability of $\beta$ lobe midline crossing was classified in a Drosophila FXS model of dfmr $1^{\Delta 3} / d f m r 1^{50 M}$ trans-heterozygotes as either normal, mild, moderate or severe. The $\beta$ lobes in $d f m r 1$ mutants cross over the midline and fuse at a fairly high frequency (93\% total, with $67 \%$ severe, $19 \%$ moderate, $7 \%$ mild midline crossing; $n=24)$. The fusion percentage of $\beta$ lobes after knock-down of NetA in dfmr1 mutant flies did not differ significantly compared with $d f m r 1$ mutants ( $94 \%$ total, with $60 \%$ severe, $25 \%$ moderate, $9 \%$ mild midline crossing; $n=30$ ). However, the fusion percentage was only $42 \%$ after knock-down of NetB in dfmr1 mutants (with $10 \%$ severe, $10 \%$ moderate, $22 \%$ mild midline crossing; $n=30$ ), indicating that reduction of NetB can significantly rescue $M B$ defects in $d f m r 1$ mutants (Fig. 4a). Interestingly, a significant increase in NetB protein level was detected in the brains of $d f m r l$ mutants with an unchanged total mRNA compared with control flies $\operatorname{NetB}^{\mathrm{tm}}$ (Fig. 4b, c).

The canonical role of FMRP is translation suppression via direct mRNA-binding [42]. We performed RNA coimmunoprecipitation in cultured HEK293 cell extracts to investigate whether FMRP directly interacts with NetB human ortholog NTN1 mRNA. As shown in Fig. 4d, FMRP was precipitated by the 1C3 anti-FMRP antibody from the immunoprecipitates (IPs), Next, we performed RT-qPCR on input, IgG and anti-FMRP $\mathrm{Ab}$ immunoprecipitated fractions to assess levels of NTN1 mRNA and control mRNAs. Notably, in comparison to the negative control IgG IPs group, NTN1 mRNA was significantly enriched in the immunoprecipitated complexes. MAP1B, a known target of FMRP, was also significantly enriched in the anti-FMRP IPs. Glyceraldehyde3-phosphate dehydrogenase (GAPDH) mRNA levels did not differ between groups, further demonstrating the specificity of the NTN1-mRNA-FMRP interaction (Fig. 4e). Next, a polyribosome profile was performed to test whether FMRP regulates NTN1 mRNA translation. We used a lymphoblastoid cell line derived from an FXS patient who harbored a 237-kb deletion encompassing the entire FMR1 and FMR1NB genes [42]. In normal lymphoblastoid cells, the samples treated by cyclohexamide resulted in the accumulation of stalled polysomes, and FMRP was distributed in every fraction. The samples treated by EDTA completely disrupted polysomes into ribosomal subunits, and FMRP was only distributed in messenger ribonucleoprotein and monosomal fractions (Additional file 3: Figure S2). We analyzed the polyribosome profile of NTN1 mRNA in normal and Fmr1 mutant lymphoblastoid cells treated by cyclohexamide. As shown in Fig. 4f, the majority of NTN1 mRNA was associated with translating polyribosomes (fraction 6-10) in both normal control and Fmr1 mutant cell extracts. Notably, there was a clear increase of NTN1 mRNA in the most actively translating polyribosomes of Fmr1 mutant cells (fractions 8-10) compared with normal cells $(* * p<0.01)$. And the NTN1 mRNA level was not altered in the $\mathrm{mRNP} /$ monosomes (fractions $1-5, p>0.05$ ) but showed a decrease in the fraction 6 and $7(* p<0.05)$ in the Fmr1 mutant cells (Two-way ANOVA). These results indicate that NTN1 translation is negatively regulated by FMRP.

Furthermore, we tested courtship-associated learning and memory ability in $d f m r 1$ mutant flies when knockdown of NetB. Learning and memory in Drosophila can be investigated experimentally by taking advantage of conditioned courtship behavior, as described previously. In conditioned courtship, courting Drosophila males perform characteristic behaviors: orienting toward the female; wing extension and vibration; licking; and attempting to copulate. Virgin females generally respond by mating, but recently mated females are unreceptive and will reject to copulate. A naïve male paired with a mated female will initially court her, but his courtship activity soon decreases. After $1 \mathrm{~h}$ of training with the mated female, his courtship activity remains depressed for 2 to $3 \mathrm{~h}$ when subsequently paired with a virgin female [24, 25]. As the results showed in Fig. 4g, dfmr1 mutants showed low courtship interest in virgin females (naïve), impaired immediate recall memory at 0-2 min post-training, and a short-term memory deficit for up to $1 \mathrm{~h}$, as previously reported [24, 25, 43]. A similar phenotype was also found during knock-down of NetA in dfmr1 mutants. However, knock-down of NetB significantly ameliorated deficits in courtship activity, immediate recall memory and short-term memory.

Considering the olfactory conditioning short-term memory was impaired in $d f m r 1$ mutants [44], we asked whether knock-down of NetB in $d f m r 1$ mutants can also ameliorate the olfactory short-term memory. $d f m r 1 \mathrm{mu}-$ tants displayed significant defects in olfactory based 3 min-memory compared with normal control group (elav-Gal4), so did knock-down of NetA in dfmrl mutants. We found that knock-down of NetB can partially rescue the short-term memory of $d f m r 1$ mutant flies (Fig. 4h). The tests of locomotor and olfactory abilities in $d f m r 1$ mutant flies were replicated, showing no defects as previously reported [44, 45]. We also noticed that knock-down of NetA or NetB in dfmr1 mutants showed no detectable changes in the locomotor and olfactory abilities (Additional file 4: Figure S3). Overall, we conclude that $N e t B$ regulates the learning and memory in the FXS Drosophila model.

\section{Discussion}

This study has revealed a novel function for the conserved axonal guidance cue NetB which is responsible 


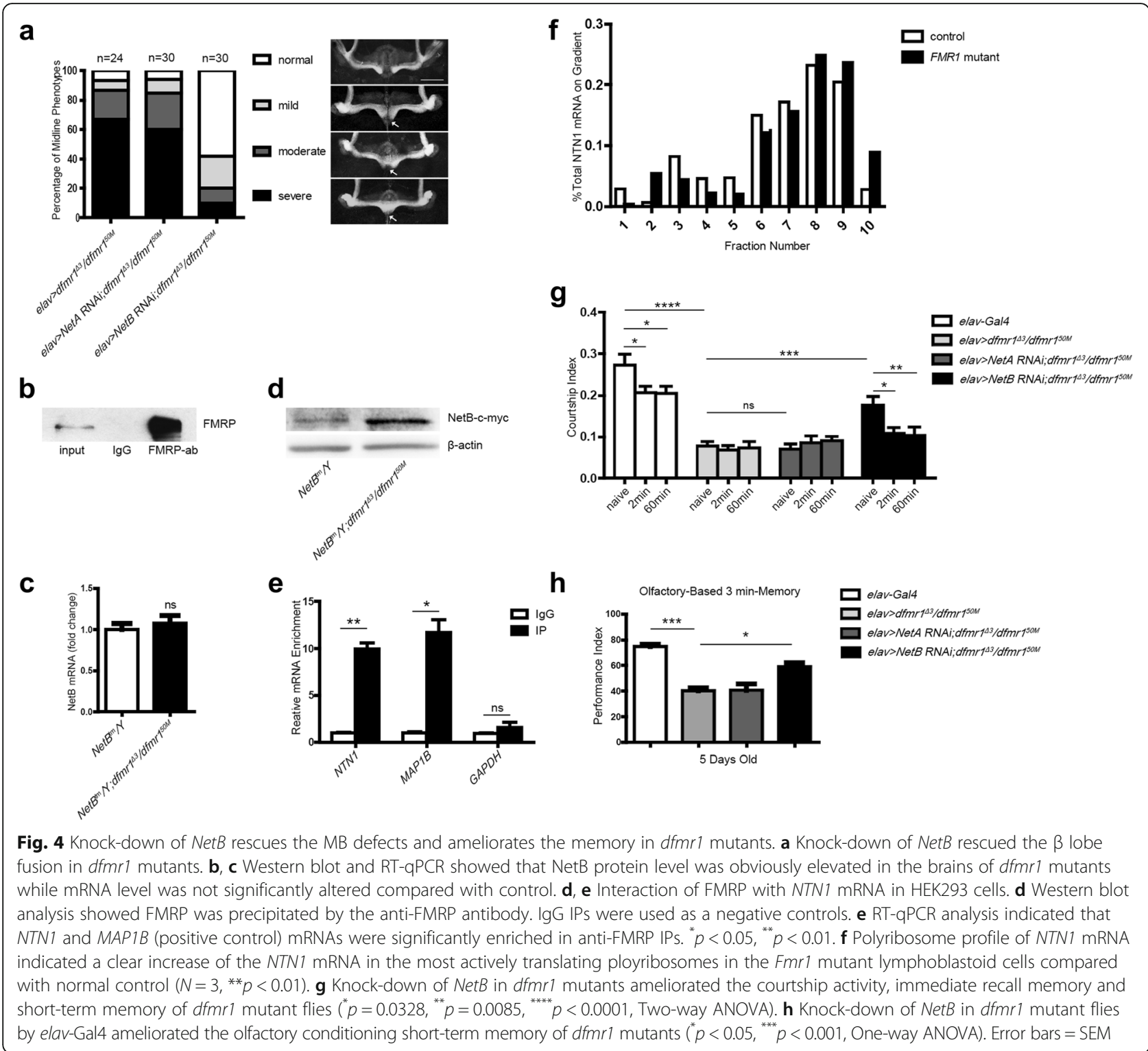

for regulating $\mathrm{MB}$ axon morphology during the development of higher-order brain centers in Drosophila. Although two Drosophila homologs of Netrin have been identified and share high homology, we demonstrate that $N e t B$, but not $N e t A$, regulates axon lobe extension of $M B$ neurons. The amino acid sequence difference in the first EGF repeat of domain $\mathrm{V}$ might alter the receptor binding specificities of NetA and NetB $[37,46,47]$, yet functional differences between NetA and NetB are still under debate. During embryonic nervous system development, NetA and NetB play similar roles in guiding commissural axon formation. When growing segmental nerve a $(\mathrm{SNa})$ motor axons are repelled outwards from the central nervous system, only NetB is required to respond to the Unc-5 receptor. While ectopic expression of NetA appears to have no effect [37, 48]. These findings imply that they may play distinct roles in mediating axon guidance. The MB is a major source of $\mathrm{NetB}$ in the developing protocerebrum, with the precise regulation of $\mathrm{MB}$ lobe extension depending on the accurate distribution of $N e t B$ expression in space and time. In the current study, NetB exhibited a specific pattern of expression in the $\mathrm{MB}$ structure just after $24 \mathrm{~h}$ APF, when $\gamma$ neuron degeneration was complete and axons had started to extend to form the mature lobes. Knock-down of NetB significantly impeded axon extensions at $27 \mathrm{~h}$ APF. These observations indicate that $N e t B$ is largely responsible for axon projection after degeneration which forms the adult-specific axon lobes.

In our study, we found that the Fra and Unc-5 receptors were involved in NetB signaling which regulates $\mathrm{MB}$ 
lobe extension, while Dscam showed no obvious genetic interaction with NetB. Dscam was involved in the mutual repulsion of sister branches, with the segregation accuracy of the sister branches depending on the repulsive force resulting from expression of the same set of Dscam isoforms. Loss of Dscam results in the failure of sister branch segregation, with flies exhibiting thin MB lobes [49]. Fra and Unc-5 affect lobe length in distinct ways, as Fra is preferential for $\alpha$ lobes, whereas Unc-5 affects the length of both $\alpha$ and $\beta$ lobes. We wonder whether NetB from the MB cells interacts with the Frazzled and Unc5 of the same cells. NetB overexpression in the MB cells showed severe $\alpha$ and $\beta$ lobe overextension, however, knock-down of Unc-5 and Fra displayed no obvious MB defects and failed to ameliorate the defects of overextended $\alpha$ or $\beta$ lobes when overexpressing NetB (Additional file 5: Figure S4a). Knock-down of Fra in glia didn't alter MB morphology, whereas knockdown of Unc-5 showed significant short MB lobes (Additional file 5: Figure S4b, $\mathrm{c}$ and Additional file 6: Table S2). These data imply that NetB from the MB cells interacts with the Unc-5 from neurons and glia surrounding the lobe fibers, and with the Fra from neurons surrounding the lobe fibers. This can be interpreted as possibly indicating a complex expression distribution of these receptors during MB development. The slit/robo2 and slit/robo3 signaling pathways provide a well-understood example of the complexity of regulation of MB lobe extension based on an intricate distribution of receptors. As Robo2 and Robo 3 proteins are expressed in many domains in the developing central complex and in fibers which cross the central neuropil, they show elaborate and distinct expression distributions in these domains across space and time. Loss-of-function mutations to each specific receptor results in different MB lobe defects [8]. Thus, further research is needed to fully understand the different processes contributing to lobe formation which depend on the NetB/Fra or NetB/Unc-5 signaling pathways.

Overexpression of $N e t B$ in the MB results in severe $\beta$ lobe fusion, resembling the defect seen in $d f m r 1 \mathrm{mu}-$ tants. As a translation suppressor binding to specific mRNAs, lack of FMRP results in dysregulated translation of many mRNAs, including those critical to neuronal development, plasticity, and dendritic spine architecture [30]. In this study, we identified NTN1 mRNA as a specific target of FMRP. NetB protein levels were significantly enhanced in the brains of $d f m r 1 \mathrm{mu}-$ tants. Knock-down of NetB significantly rescued $d f m r 1$-related MB defects and ameliorated deficits in short-term learning and memory in FXS model Drosophila. As a conserved axon guidance cue, NTN1 directs synaptic formation and synaptic plasticity during development [50].
Restricted sources of NTN1 can regulate the rapid local recruitment of synaptic proteins during neural development and spatially mediate the local translation of the synaptic proteome in Aplysia neurons for synaptic consolidation [50-53]. In the mature mammalian brain, NTN1 and its receptor DCC (an ortholog of Fra) are enriched at synapses, disruption of NTN1/DCC signaling reduces dendritic spine volume, severely attenuates LTP at hippocampal Schaffer collateral synapses, and impairs hippocampal-dependent learning and memory [54]. These findings suggest that overexpression of NTN1 leads to synaptic structural defects, which may contribute to cognitive impairment in FXS.

In summary, we provide evidence for a significant regulation of NetB signaling in the MB lobe extension. We describe a novel FMRP target and find that knock-down of $N e t B$ can partially restore learning and memory in $d f m r 1$ mutants.

\section{Additional files}

Additional file 1: Table S1. NetB affects $\alpha^{\prime} / \beta^{\prime}$ and $\gamma$ lobe length (Fisher exact test). (DOCX $15 \mathrm{~kb}$ )

Additional file 2: Figure S1. a-c. Knock-down of Netrins with panneuronal driver elav-Gal4 showed similar phenotypes as driven by OK107Gal4. a. MBs from control (elav-Gal4 > mCD8-GFP), NetA RNAi, NetB RNAi, NetA OE and NetB OE were visulized by $m$ CD8-GFP driven by elav-Gal4 and immunostaining for Fasll. The Control, NetA RNAi, NetA OE MBs showed normal structures, but knock-down of NetB showed short lobes (arrowhead). NetB OE MBs showed overextended lobes (arrow). b. The percentage of brain hemispheres with short $\alpha / \beta$ lobes in NetA/NetB RNAi flies. (elav-Gal4, $n=35$; elav > NetA RNAi, $n=40$; elav > NetB RNAi, $n=30$; $\left.{ }^{* * *} p<0.001\right)$. c. The percentage of brain hemispheres with overextension of $\alpha / \beta$ lobes in flies over-expressing Netrins. (elav-Gal4, $n=35$; elav $>$ UAS NetA, $n=30$; elav $>$ UAS-NetB, $n=30,{ }^{* * *} p<0.001$ ). Significance was determined by Fisher exact test. Scale bars: 50 m. (TIF 6225 kb)

Additional file 3: Figure S2. The distribution of FMRP in the normal lymphoblastoid cell extracts treated with cycloheximide and EDTA. After cycloheximide treatment, FMRP was distributed across all the fractions. However, in the EDTA treated samples, most of the FMRP was in fractions $1-5$, which correspond to the free messenger ribonucleoprotein (mRNP) and monosomal fraction. (TIF $685 \mathrm{~kb}$ )

Additional file 4: Figure S3. Analysis of locomotor and olfactory abilities. a. Locomotor activity was measured by a line crossing assay [34]. All genotypes had similar locomotor activity profiles (elav-Gal4/Y, elavGal4/Y;dfmr1 ${ }^{\Delta 3} / d f m r 1^{50 M}$, elav-Gal4/Y; UAS-NetA RNAi/ $+; d f m r 1^{\Delta 3} / d f m r 1^{50 M}$, elav-Gal4/Y; UAS-NetB RNAi/+; dfmr1 ${ }^{\Delta 3} / d f m r 1^{50 M}$, for each genotype, we tested 20 flies). b. Olfactory capabilities were measured by the olfactory trap assay [35]. No differences were found between any of the genotypes tested with this assay at the $60 \mathrm{~h}$ time point. (TIF 6938 kb)

Additional file 5: Figure S4. NetB from the MB cells doesn't interact with the Fra and Unc5 of the same cells. a. Knock-down of Fra and Unc-5 with NetB overexpression in the MB cells failed to ameliorate the defects of overextended $a$ and $\beta$ lobes (OK107 > UAS-NetB, $n=28$; UAS-NetB/+; UAS-Fra RNAi/+; OK107-Gal4/+, $n=30$; UAS-NetB/+; UAS-Unc-5 RNAi/+; OK107-Gal4/+, $n=28$ ). b. Knock-down of Fra and Unc-5 with OK107-Gal4 displayed normal $\alpha / \beta$ lobes with normal length. Knock-down of Fra by a glia specific driver repo-Gal4, the MBs showed normal structure. However, knock-down of Unc-5 with repo-Gal4 caused short a/ $\beta$ lobes (arrowhead), c. The percentage of brain hemispheres with short a/ $\beta$ lobes (OK107-Gal4, $n=30$; repo-Gal4, $n=28$; OK107 > Fra RNAi, $n=25$, OK107 > Unc-5 RNAi, $n=20$; repo $>$ Fra RNAi, $n=20$; repo $>$ Unc-5 RNAi, $n=22, \quad p<0.001$ ). 
Significance was determined by Fisher's exact test. Scale bars: 50 um. (TIF $5078 \mathrm{~kb}$ )

Additional file 6: Table S2. The summary of the $\alpha / \beta$ lobe defects in knocking-down Fra or Unc-5 by pan-neuron, MB and glia drivers respectively. (DOCX $15 \mathrm{~kb})$

\section{Abbreviations}

APF: After pupal formation; Cl: Courtship index; Dscam: Down syndrome cell adhesion molecule; EB: Ellipsoid body; Fmr1: Fragile-X mental retardation 1; FMRP: Fragile-X mental retardation protein; Fra: Frazzled; FXS: Fragile $X$ syndrome; GAPDH: Glyceraldehyde-3-phosphate dehydrogenase; IPS: Immunoprecipitates; MAP1B: Micro-tubule associated protein; MB: Mushroom body; NetA: Netrin-A; NetB: Netrin-B; NTN1: Netrin-1; RNA- IP: RNA immunoprecipitation; SNa: Segmental nerve a; Unc5: Uncoordinated-5; Unc-5: Uncoordinated-5; VNC: ventral nerve cord

\section{Acknowledgements}

The authors thank H, Qin (College of Biology, Hunan University) for his help in the test of olfactory conditioning paradigm.

\section{Funding}

This study was supported by the National Natural Science Foundation of China (grant number 81571253,81771385 ) and the Hunan Provincial Natural Science Foundation of China (grant number 2016JJ3135).

\section{Availability of data and materials}

Data sharing not applicable to this article as no datasets were generated or analyzed during the current study.

\section{Authors' contributions}

$\mathrm{RD}$ and HC designed research; HK, JZ and RD wrote the article; HK, JZ, XJ, GL, WH performed research; HK analyzed data. All authors read and approved the final manuscript.

\section{Ethics approval and consent to participate}

Written informed consent was obtained from parents and patients older than age 18 years for participation in this study, which was approved by the ethics committee of the state key laboratory of medical genetics, Central South University (approval number: 2013051201)

\section{Consent for publication}

Not applicable.

\section{Competing interests}

The authors declare that they have no competing interests.

\section{Publisher's Note}

Springer Nature remains neutral with regard to jurisdictional claims in published maps and institutional affiliations.

\section{Author details}

${ }^{1}$ Center for Medical Genetics, School of Life Sciences, Central South University, Changsha 410078, Hunan, China. ${ }^{2}$ Changchun Children' Hospital, Changchun 130000, Jilin, China. ${ }^{3}$ Hunan Key Laboratory of Medical Genetics, Central South University, Changsha 410078, Hunan, China. ${ }^{4}$ Hunan Key Laboratory of Animal Models for Human Diseases, Central South University, Changsha 410078, Hunan, China.

Received: 27 December 2018 Accepted: 6 May 2019

Published online: 28 May 2019

\section{References}

1. Dickson BJ. Molecular mechanisms of axon guidance. Science. 2002 298(5600):1959-64.

2. Lowery LA, Van Vactor D. The trip of the tip: understanding the growth cone machinery. Nat Rev Mol Cell Biol. 2009;10(5):332.

3. Oliva C, Soldano A, Mora N, De Geest N, Claeys A, Erfurth ML, et al. Regulation of Drosophila brain wiring by neuropil interactions via a slit-Robo-RPTP signaling complex. Dev Cell. 2016;39(2):267-78.
4. Boyle M, Nighorn A, Thomas JB. Drosophila Eph receptor guides specific axon branches of mushroom body neurons. Development. 2006;133(9): 1845-54.

5. Shin JE, Diantonio A. Highwire regulates guidance of sister axons in the Drosophila mushroom body. J Neurosci. 31(48):17689-700.

6. Zwarts L, Goossens T, Clements J, Kang YY, Callaerts P. Axon branch-specific Semaphorin-1a signaling in DrosophilaMushroom body development. Front Cell Neurosci. 2016;10(29):1-11.

7. Marchetti G, Reichardt I, Knoblich JA, Besse F. The TRIM-NHL protein brat promotes axon maintenance by repressing src64B expression. J Neurosci. 2014;34(41):13855-64

8. Nicolas E, Preat T. Drosophila central brain formation requires Robo proteins. Dev Genes Evol. 2005:215(10):530-6.

9. Serafini T, Colamarino SA, Leonardo ED, Wang H, Beddington R, Skarnes WC et al. Netrin-1 is required for commissural axon guidance in the developing vertebrate nervous system. Cell. 1996;87(6):1001.

10. Lai Wing Sun K, Correia JP, Kennedy TE. Netrins: versatile extracellular cues with diverse functions. Development. 2011;138(11):2153.

11. Kolodziej PA, Timpe LC, Mitchell KJ, Fried SR, Goodman CS, Jan LY, et al Frazzled encodes a Drosophila member of the DCC immunoglobulin subfamily and is required for CNS and motor axon guidance. Cell. 1996: 87(2):197-204

12. Hiramoto $M$, Hiromi $Y$, Giniger $E$, Hotta $Y$. The Drosophila netrin receptor frazzled guides axons by controlling netrin distribution. Nature. 2000; 406(6798):886-9.

13. Keleman K, Dickson BJ. Short- and long-range repulsion by the Drosophila Unc5 netrin receptor. Neuron. 2001;32(4):605.

14. Lewis TL Jr, Courchet J, Polleux F. Cellular and molecular mechanisms underlying axon formation, growth, and branching. J Cell Biol. 2013;202(6): 837-48

15. Hattori D, Demir E, Kim HW, Viragh E, Zipursky SL, Dickson BJ. Dscam diversity is essential for neuronal wiring and self-recognition. Nature. 2007; 449(7159):223.

16. Goyal G, Zheng J, Adam E, Steffes G, Jain M, Klavins K, et al. Sphingolipiddependent Dscam sorting regulates axon segregation. Nat Commun. 2019; 10(813):1-17.

17. Zhan XL, Clemens JC, Neves G, Hattori D, Flanagan JJ, Hummel T, et al. Analysis of Dscam diversity in regulating axon quidance in Drosophila mushroom bodies. Neuron. 2004;43(5):673-86.

18. Fiala A. Olfaction and olfactory learning in Drosophila: recent progress. Curr Opin Neurobiol. 2007;17(6):720-6.

19. Davis RL. Traces of Drosophila memory. Neuron. 2011;70(1):8-19.

20. Kahsai L, Zars T. Learning and memory in Drosophila: behavior, genetics, and neural systems. Int Rev Neurobiol. 2011;99:139.

21. Waddell S. Reinforcement signalling in Drosophila ; dopamine does it all after all. Curr Opin Neurobiol. 2013;23(3):324-9.

22. Bushey D, Cirelli C. From genetics to structure to function: exploring sleep in Drosophila. Int Rev Neurobiol. 2011;99(24):213

23. Drozd M, Bardoni B, Capovilla M. Modeling Fragile X Syndrome in Drosophila. Front Mol Neurosci. 2018;11(124):1-15

24. Mcbride SM, Choi CH, Wang Y, Liebelt D, Braunstein E, Ferreiro D, et al. Pharmacological Rescue of Synaptic Plasticity, courtship behavior, and mushroom body defects in a Drosophila model of fragile X syndrome. Neuron. 2005:45(5):753-64.

25. Chang S, Bray SM, Li Z, Zarnescu DC, He C, Jin P, et al. Identification of small molecules rescuing fragile $X$ syndrome phenotypes in Drosophila. Nat Chem Biol. 2008:4(4):256.

26. Chakraborty R, Vepuri V, Mhatre SD, Paddock BE, Miller S, Michelson SJ, et al. Characterization of a Drosophila Alzheimer's disease model: pharmacological Rescue of Cognitive Defects. PLoS One. 2011;6(6):e20799.

27. Kelly SM, Bienkowski R, Banerjee A, Melicharek DJ, Brewer ZA, Marenda DR, et al. The Drosophila ortholog of the $\mathrm{ZC} 3 \mathrm{H} 14$ RNA binding protein acts within neurons to pattern axon projection in the developing brain. Dev Neurobiol. 2016:76(1):93-106.

28. Santoro MR, Bray SM, Warren ST. Molecular mechanisms of fragile $X$ syndrome: a twenty-year perspective. Annu Rev Pathol. 2011:7(1):219-45.

29. Jacquemont S, Hagerman RJ, Hagerman PJ, Leehey MA. Fragile-X syndrome and fragile $X$-associated tremor/ataxia syndrome: two faces of FMR1. Lancet Neurol. 2007;6(1):45-55.

30. Davis JK, Broadie K. Multifarious functions of the fragile X mental retardation protein. Trends Genet. 2017;33(10):703. 
31. Rollmann SM, Zwarts L, Edwards AC, Yamamoto A, Callaerts P, Norga K, et al. Pleiotropic effects of Drosophila neuralized on complex behaviors and brain structure. Genetics. 2008;179(3):1327-36.

32. Gross C, Nakamoto M, Yao X, Chan CB, Yim SY, Ye K, et al. Excess phosphoinositide 3-kinase subunit synthesis and activity as a novel therapeutic target in fragile X syndrome. J Neurosci. 2010;30(32):10624.

33. Lu R, Wang H, Liang Z, Ku L, O'Donnell WT, Li W, et al. The fragile X protein controls microtubule-associated protein $1 \mathrm{~B}$ translation and microtubule stability in brain neuron development. Proc Natl Acad Sci U S A. 2004; 101(42):15201-6

34. Siegel RW, Hall JC. Conditioned responses in courtship behavior of normal and mutant Drosophila. Proc Natl Acad Sci U S A. 1979;76(7):3430-4.

35. Mcbride SM, Giuliani G, Choi C, Krause P, Correale D, Watson K, et al. Mushroom body ablation impairs short-term memory and long-term memory of courtship conditioning in Drosophila melanogaster. Neuron. 1999;24(4):967-77.

36. Tully T, Preat T, Boynton SC, Del Vecchio M. Genetic dissection of consolidated memory in Drosophila. Cell. 1994;79(1):35-47.

37. Brankatschk M, Dickson BJ. Netrins guide Drosophila commissural axons at short range. Nat Neurosci. 2006;9(2):188.

38. Orr BO, Borgen MA, Caruccio PM, Murphey RK. Netrin and frazzled regulate presynaptic gap junctions at a Drosophila giant synapse. J Neurosci. 2014; 34(16):5416-30.

39. Lee T, Lee A, Luo L. Development of the Drosophila mushroom bodies: sequential generation of three distinct types of neurons from a neuroblast. Development. 1999:126(18):4065-76.

40. Andrews GL, Tanglao S, Farmer WT, Morin S, Brotman S, Berberoglu MA, et al. Dscam guides embryonic axons by netrin-dependent and -independent functions. Development. 2008;135(23):3839-48.

41. Michel $\mathrm{Cl}$, Kraft R, Restifo LL. Defective neuronal development in the mushroom bodies of Drosophila fragile $\mathrm{X}$ mental retardation 1 mutants. J Neurosci. 2004;24(25):5798-809.

42. Luo S, Huang W, Xia Q, Du Q, Wu L, Duan R. Mutational analyses of the FMR1 Gene in Chinese pediatric population of fragile X suspects: low tolerance for point mutation. J Child Neurol. 2015;30(6):803-6.

43. Monyak RE, Emerson D, Schoenfeld BP, Zheng X, Chambers DB, Rosenfelt C, et al. Insulin signaling misregulation underlies circadian and cognitive deficits in a Drosophila fragile X model. Mol Psychiatry. 2016;22(8):1140-8.

44. Bolduc FV, Bell K, Cox H, Broadie KS, Tully T. Excess protein synthesis in Drosophila fragile $\mathrm{X}$ mutants impairs long-term memory. Nat Neurosci. 2008;11(10):1143-5.

45. Dockendorff TC, Su HS, Mcbride SM, Yang Z, Choi CH, Siwicki KK, et al. Drosophila lacking dfmr1 activity show defects in circadian output and fail to maintain courtship interest. Neuron. 2002:34(6):973-84.

46. Mitchell KJ, Doyle JL, Serafini T, Kennedy TE, Tessier-Lavigne M, Goodman CS, et al. Genetic analysis of netrin genes in Drosophila: netrins guide CNS commissural axons and peripheral motor axons. Neuron. 1996;17(2):203-15.

47. Harris R, Sabatelli LM, Seeger MA. Guidance cues at the Drosophila CNS midline: identification and characterization of two Drosophila netrin/UNC-6 homologs. Neuron. 1996;17(2):217.

48. Newquist G, Drennan JM, Lamanuzzi M, Walker K, Clemens JC, Kidd T. Blocking apoptotic signaling rescues axon guidance in netrin mutants. Cell Rep. 2013;3(3):595-606

49. Wang J, Zugates $C T$, Liang $\mathbb{H}_{\text {, Lee }} \mathrm{CH}$, Lee T. Drosophila Dscam is required for divergent segregation of sister branches and suppresses ectopic bifurcation of axons. Neuron. 2002;33(4):559-71.

50. Goldman JS, Ashour MA, Magdesian MH, Tristsch NX, Harris SN, Christofi N, et al. Netrin-1 promotes excitatory synaptogenesis between cortical neurons by initiating synapse assembly. J Neurosci. 2013;33(44):17278-89.

51. Colón-Ramos DA, Margeta MA, Shen K. Glia promote local synaptogenesis through UNC-6 (netrin) signaling in C. elegans. Science. 2007:318(5847):103-6.

52. Poon W, Klassen MP, Shen K. UNC-6/netrin and its receptor UNC-5 locally exclude presynaptic components from dendrites. Nature. 2008:455(7213):669-73.

53. Kim S, Martin KC. Neuron-wide RNA transport combines with netrin-mediated local translation to spatially regulate the synaptic proteome. eLife. 2015;4(201501-08):1-24.

54. Horn KE, Glasgow SD, Gobert D, Bull SJ, Luk T, Girgis J, et al. DCC expression by neurons regulates synaptic plasticity in the adult brain. Cell Rep. 2013; 3(1):173-85.

\section{Ready to submit your research? Choose BMC and benefit from:}

- fast, convenient online submission

- thorough peer review by experienced researchers in your field

- rapid publication on acceptance

- support for research data, including large and complex data types

- gold Open Access which fosters wider collaboration and increased citations

- maximum visibility for your research: over $100 \mathrm{M}$ website views per year

At BMC, research is always in progress.

Learn more biomedcentral.com/submissions 